\title{
SOLVING BINOMIAL THUE EQUATIONS
}

\section{István Gaál and László Remete}

Mathematical Institute

University of Debrecen

H-4010 Debrecen Pf.12, Hungary

e-mail: igaal@science.unideb.hu

remete142@gmail.com

\begin{abstract}
We consider binomial Thue equations of type $x^{n}-m y^{n}= \pm 1$ in $x, y \in \mathbb{Z}$. Optimizing the method of Pethö [7] we perform an extensive calculation by a high performance computer to determine all solutions with $\max (|x|,|y|)<10^{500}$ of binomial Thue equations for $m<10^{7}$ for exponents $n=3,4,5,7,11,13,17,19,23,29$.
\end{abstract}

\section{Introduction}

The method of Pethő [7] (see also [4]) gives a fast algorithm to calculate "small" solutions of Thue equations. The method is based on the continued fraction algorithm. By "small" solutions we mean those with absolute values Received: October 4, 2014; Accepted: November 12, 2014 2010 Mathematics Subject Classification: Primary 11D59; Secondary 11 Y50.

Keywords and phrases: binomial Thue equations, supercomputers.

This work was partially supported by the European Union and the European Social Fund through project Supercomputer, the national virtual lab (grant no.: TAMOP-4.2.2.C11/1/KONV-2012-0010) and also supported in part by K100339 and K75566 from the Hungarian National Foundation for Scientific Research.

Communicated by K. K. Azad 
less than, say $10^{500}$. Nobody believes that such equations have larger solutions.

All our experiences show that such equations usually only have a few very small solutions.

In this paper, we make this algorithm more efficient in order to calculate "small" solutions of a special type of Thue equations, the binomial Thue equations of type

$$
x^{n}-m y^{n}= \pm 1 \text { in } x, y \in \mathbb{Z} .
$$

Using sharp estimates and utilizing the specialties of these equations we perform an extensive calculation by a high performance computer to determine solutions with $\max (|x|,|y|)<10^{500}$ of binomial Thue equations for $1<m<10^{7}$ (assuming that the left hand side is irreducible) for the exponents $n=3,4,5,7,11,13,17,19,23,29$. Our data contains all solutions of these equations with high probability. These results complete several results on the solutions of binomial Thue equations [2], [1], [6].

\section{Sharper Estimates}

Let $n$ be one of 3, 5, 7, 11,13,17, 19, 23, 29, in fact our arguments are valid for any odd primes (the case $n=4$ we shall deal with later). Assume that for $m$ the left hand side of (1) is irreducible (we skip those $m$ for which the left hand side of (1) is reducible). Our purpose is to determine all solutions $x, y \in \mathbb{Z}$ of (1) with $\max (|x|,|y|)<C$. We shall perform our calculation with $C=10^{500}$.

Let $\zeta=\exp (2 \pi i / n)$. Let $x, y$ be an arbitrary solution of (1). Set

$$
\beta_{j}=x-\zeta^{j-1} \sqrt[n]{m} y
$$

for $j=1, \ldots, n$, then equation (1) can be written as

$$
\beta_{1} \cdots \beta_{n}= \pm 1
$$


We may assume $y \geq 0$ since on the right side we have \pm 1 in our equation. Also, for $y=0$ we only have the trivial solution $x= \pm 1$. Therefore in the following let $y \geq 1$.

For $n \neq 4$ the $\beta_{2}, \ldots, \beta_{n}$ are complex, therefore

$$
\left|\beta_{j}\right|=\left|x-\zeta^{j-1} \sqrt[n]{m} y\right| \geq\left|\operatorname{Im}\left(\zeta^{j-1}\right)\right| \sqrt[n]{m} y
$$

for $j=1, \ldots, n$. This yields that

$$
\left|\beta_{1}\right|=|x-\sqrt[n]{m} y| \leq \frac{1}{c_{1} y^{n-1}}
$$

that is

$$
\left|\sqrt[n]{m}-\frac{x}{y}\right| \leq \frac{1}{c_{1} y^{n}}
$$

with

$$
c_{1}=(\sqrt[n]{m})^{n-1} c_{2}, \text { where } c_{2}=\prod_{j=2}^{n}\left|\operatorname{Im}\left(\zeta^{j-1}\right)\right|
$$

Thus, we arrived at the crucial point of the method of [7]. Obviously $(x, y)=1$. If the upper estimate on the right hand side of (3) satisfies

$$
\frac{1}{c_{1} y^{n}}<\frac{1}{2 y^{2}}
$$

that is

$$
y>\left(2 c_{1}\right)^{1 /(n-2)},
$$

then (3) implies

$$
\left|\sqrt[n]{m}-\frac{x}{y}\right|<\frac{1}{2 y^{2}}
$$


and appling Legenre's theorem we confer that $x / y$ is a convergent $h_{j} / k_{j}$ to $\sqrt[n]{m}$, that is $x=h_{j}, y=k_{j}$. For the corresponding partial quotients $a_{j}$ it is well known that

$$
\frac{1}{\left(a_{j+1}+2\right) k_{j}^{2}}<\left|\sqrt[n]{m}-\frac{x}{y}\right|
$$

Let $A=\max _{1 \leq i \leq s} a_{i}$, where $k_{s}$ is the first denominator of a partial quotient exceeding $C$. Combining the above estimate with (3) we get

$$
\frac{1}{(A+2) k_{j}^{2}}<\left|\sqrt[n]{m}-\frac{x}{y}\right| \leq \frac{1}{c_{1} k_{j}^{n}}
$$

whence we obtain

$$
y=k_{j}<c_{3}=\left(c_{1}(A+2)\right)^{n-2} .
$$

We calculate all denominators of partial quotients up to $C$, we take their maximum, calculate the above bound and check all possible $x=h_{j}, y=k_{j}$ for $k_{j}<c_{3}$ running again the continued fraction algorithm.

Now we return to the validity of (4). Simple calculation shows that (4) is satisfied if

$$
y>\left(\frac{2}{c_{2}}\right)^{\frac{1}{n-2}} m^{-\frac{n-1}{n(n-2)}} .
$$

If $m$ is large enough, then condition (5) is satisfied for $y \geq 1$. Some small values of $y$ must be tested for the following values of $m$ : 


\begin{tabular}{|c|c|}
\hline$n$ & \\
\hline 3 & $2 \leq m \leq 4$ \\
\hline 5 & $2 \leq m \leq 10$ \\
\hline 7 & $2 \leq m \leq 29$ \\
\hline 11 & $2 \leq m \leq 314$ \\
\hline 13 & $2 \leq m \leq 1078$ \\
\hline 17 & $2 \leq m \leq 13489$ \\
\hline 19 & $2 \leq m \leq 48699$ \\
\hline 23 & $2 \leq m \leq 652798$ \\
\hline 29 & $2 \leq m \leq 7960210$ \\
\hline
\end{tabular}

For all these values of $m$ we have to test all $y$ with

$$
y \leq\left(\frac{2}{c_{2}}\right)^{\frac{1}{n-2}} m^{-\frac{n-1}{n(n-2)}} .
$$

Easy calculation shows that this merely yields testing $y=1$ for the values of $m$ contained in the table. This can be done very fast.

Remark. The case $n=4$ was considered in [5]. Remark that in that case we may assume $x>0, y>0$. We have

$$
|x-\sqrt[4]{m} y| \leq 1, \quad|x \pm i \sqrt[4]{m} y| \geq \sqrt[4]{m} y
$$

therefore

$$
|x+\sqrt[4]{m} y| \geq 2 \sqrt[4]{m} y-1 \geq \sqrt[4]{m} y
$$

whence

$$
\left|\sqrt[4]{m}-\frac{x}{y}\right| \leq \frac{1}{(\sqrt[4]{m})^{3}} \frac{1}{y^{4}}<\frac{1}{2 y^{2}}
$$

where the last inequality is valid for all $y \geq 1$. 


\section{Computational Aspects}

We were executing the algorithm of [7] with $C=10^{500}$ for $n=3,4,5$, $7,11,13,17,19,23,29$ (the case $n=4$ was dealt with in [5]) using the above sharp estimates that made the procedure for binomial Thue equations much more efficient. This efficient algorithm allowed us to perform the calculations for all those $2 \leq m \leq 10^{7}$ for which the left side of (1) is irreducible.

The procedure was implemented in Maple [3], we used 1200 digits accuracy. For each exponent this calculation involved almost $10^{7}$ binomial Thue equations. The routines were running on the supercomputer (high performance computer) network situated in Debrecen-Budapest-Pécs-Szeged in Hungary under Linux. For each exponent the total running time was about 120-200 hours calculated for a single node which yields a few hours using parallel computing with a couple of nodes.

\section{Solutions}

In this chapter, we list the results of our computation. The triples $(m, x, y)$ in our table mean that for the $m$ there is a solution $x, y$ of equation (1). The trivial solution $(m, x, y)=(m, 1,0)$ is not listed. The solutions are displayed up to sign, that is we include only one of $(m, x, y)$ and $(m,-x,-y)$.

For each exponent $m$, we list the solutions with

$$
\max (|x|,|y|)<10^{500}
$$

of all equations with $2 \leq m \leq 10^{7}$ for which the left hand side of equation (1) is irreducible. 
4.1. Solutions for $n=3$

\begin{tabular}{|c|c|c|c|c|c|c|c|c|c|c|c|c|c|c|}
\hline$m$ & $x$ & y & $m$ & 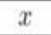 & 9 & $m$ & $x$ & $y$ & $m$ & 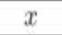 & $y$ & $m$ & $x$ & $y$ \\
\hline 2 & 1 & 1 & 3155 & 44 & 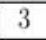 & 8745 & 1036 & 39 & 54873 & 38 & 1 & 117650 & 49 & 1 \\
\hline 7 & 2 & 1 & 74 & 15 & 1 & 963 & 80 & 3 & 7811 & 116 & 3 & 18681 & 344 & -7 \\
\hline 9 & 2 & 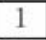 & 76 & 5 & 1 & 441 & 242 & & 9318 & 39 & 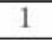 & 23506 & 249 & 5 \\
\hline 17 & 18 & 7 & 3605 & 46 & 3 & 9682 & 27 & 1 & 59320 & 39 & 1 & 124999 & 50 & 7 \\
\hline 19 & 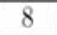 & 3 & 724 & 31 & 2 & 9684 & 27 & 7 & 60853 & 118 & 2 & 25001 & 50 & 1 \\
\hline 20 & 19 & 7 & 3907 & 63 & 4 & 19927 & 244 & 9 & 1630 & 79 & 2 & 126506 & 251 & 5 \\
\hline 26 & 3 & $\overline{1}$ & 4095 & 16 & 1 & 20421 & 82 & 3 & 63999 & $\overline{40}$ & 1 & 130067 & 152 & 3 \\
\hline 28 & 3 & 1 & 4097 & 16 & 1 & 20797 & 55 & 8 & 64001 & 40 & 1 & 132650 & 51 & 1 \\
\hline 37 & 10 & 3 & 4291 & 65 & 4 & 21951 & 28 & - & 66430 & 81 & 2 & 132652 & 51 & 1 \\
\hline 43 & 7 & 2 & 4492 & 33 & 2 & 21953 & 28 & 1 & 68920 & 41 & 1 & 135269 & 154 & 3 \\
\hline 63 & 4 & 1 & 4912 & 17 & 1 & 23149 & 57 & 2 & 68922 & 41 & 1 & 136591 & 103 & 2 \\
\hline 65 & 4 & 1 & 4914 & 17 & 1 & 24388 & 29 & 1 & 72338 & 125 & 3 & 137160 & 361 & 7 \\
\hline 91 & 9 & 2 & 5080 & 361 & 21 & 24390 & 29 & 1 & 74087 & 42 & 1 & 138303 & 362 & 7 \\
\hline 124 & 5 & 1 & 5514 & 53 & 3 & 26110 & 89 & 3 & 74089 & 42 & 1 & 140607 & 52 & 1 \\
\hline 126 & 5 & 1 & 5831 & 18 & 1 & 26999 & 30 & 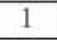 & 75866 & 127 & 3 & 140609 & 52 & 7 \\
\hline 182 & 17 & 3 & 5833 & 18 & 1 & 27001 & 30 & 1 & 79506 & 43 & 1 & 144703 & 105 & 2 \\
\hline 215 & 6 & 1 & 6162 & 55 & 3 & 27910 & 91 & 3 & 79508 & 43 & 1 & 148876 & 53 & 1 \\
\hline 217 & 6 & 1 & $\begin{array}{l}6858 \\
\end{array}$ & 19 & 1 & 29790 & 31 & 1 & 82313 & 87 & 2 & 148878 & 53 & 1 \\
\hline 254 & 19 & 3 & 6860 & 19 & 1 & 29792 & 31 & 1 & 85183 & 44 & 1 & 154566 & 161 & 3 \\
\hline 342 & 7 & 1 & 7415 & 39 & 2 & 31256 & 63 & 2 & 85185 & 44 & 1 & 156494 & 485 & 9 \\
\hline 344 & 7 & 1 & 7999 & 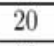 & 1 & 32006 & 127 & 4 & 87866 & 9825 & 221 & 157463 & 54 & 1 \\
\hline 422 & 15 & 2 & 8001 & 20 & 1 & 32042 & 667 & 21 & 88121 & 89 & 2 & 157465 & 54 & 1 \\
\hline 511 & 8 & 1 & 8615 & 41 & 2 & 32767 & 32 & 1 & 89115 & 134 & 3 & 158438 & 487 & 9 \\
\hline 513 & 8 & 1 & 8827 & 62 & 3 & 32769 & 32 & 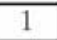 & 91124 & 45 & 1 & 160398 & 163 & 3 \\
\hline 614 & 17 & 2 & 9260 & 21 & 1 & 33542 & 129 & 4 & 91126 & 45 & 1 & 166374 & 55 & 1 \\
\hline 635 & 361 & 42 & 9262 & 21 & 1 & 34328 & 65 & 2 & 93165 & 136 & 3 & 166376 & 55 & 1 \\
\hline 651 & 26 & 3 & 9709 & 64 & 3 & 34859 & 98 & 3 & 97335 & 46 & 1 & 170954 & 111 & 2 \\
\hline 728 & 0 & 1 & 10647 & 22 & 1 & 35936 & 33 & 1 & 97337 & 46 & 1 & 175615 & 56 & 1 \\
\hline 730 & 9 & 1 & 10649 & 22 & 1 & 35938 & 33 & 1 & 99161 & 324 & 7 & 175617 & 56 & 1 \\
\hline 813 & 28 & 3 & 12166 & 23 & 1 & 37037 & 100 & 3 & 100082 & 325 & 7 & 180362 & 113 & 2 \\
\hline 999 & 10 & 1 & 12168 & 23 & 1 & 39303 & 34 & 1 & 103822 & 47 & 7 & 181963 & 170 & 3 \\
\hline 1001 & 10 & 1 & 12978 & 47 & 2 & 39305 & 34 & 1 & 103824 & 47 & 1 & 185192 & 57 & 1 \\
\hline 1330 & 11 & 1 & 13256 & 71 & 3 & 42874 & 35 & 1 & 107172 & 95 & 2 & 185194 & 57 & 1 \\
\hline 1332 & 11 & 1 & 13538 & 143 & 6 & 42876 & 35 & 1 & 108304 & 143 & 3 & 188461 & 172 & 3 \\
\hline 1521 & 23 & 2 & 13823 & 24 & 1 & 44739 & 71 & 2 & 108873 & 191 & 4 & 195111 & 58 & 1 \\
\hline 1588 & 35 & 3 & 13825 & 24 & 1 & 45372 & 107 & 3 & 109444 & 287 & 6 & 195113 & 58 & 1 \\
\hline 1657 & 71 & 6 & 14114 & 145 & 6 & 46011 & 215 & 6 & 110017 & 575 & 12 & 205378 & 59 & 1 \\
\hline 1727 & 12 & 1 & 14408 & 73 & 3 & 46655 & 36 & 1 & 110591 & 48 & 1 & 205380 & 59 & 1 \\
\hline 1729 & 12 & 1 & 14706 & 49 & 2 & 46657 & 36 & 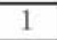 & 110593 & 48 & 1 & 210645 & 119 & 2 \\
\hline 1801 & 73 & 6 & 15253 & 124 & 5 & 47307 & 217 & 6 & 111169 & 577 & 12 & 212420 & 179 & 3 \\
\hline 1876 & 37 & 3 & 15624 & 25 & 1 & 47964 & 109 & 3 & 111748 & 289 & 6 & 214205 & 359 & 6 \\
\hline 1953 & 25 & 2 & 15626 & 25 & 1 & 48627 & 73 & 2 & 112329 & 193 & 4 & 215999 & 60 & 1 \\
\hline 2196 & 13 & 1 & 16003 & 126 & 5 & 48949 & 4097 & 112 & 112912 & 145 & 3 & 216001 & 60 & 1 \\
\hline 2198 & 13 & 1 & 17145 & 361 & 14 & 50652 & 37 & 1 & 114084 & 97 & 2 & 217805 & 361 & 6 \\
\hline 2743 & 14 & 1 & 17575 & 26 & 1 & 50654 & 37 & 1 & 116623 & 342 & 7 & 219620 & 181 & 3 \\
\hline 2745 & 14 & 1 & 17577 & 26 & 1 & 54871 & 38 & 1 & 117648 & 49 & 1 & 221445 & 121 & , \\
\hline
\end{tabular}




\begin{tabular}{|c|c|c|c|c|c|c|c|c|c|c|c|c|c|c|}
\hline$m$ & $x$ & $y$ & $m$ & $x$ & $y$ & $m$ & $x$ & $y$ & $m$ & $x$ & $y$ & $m$ & $x$ & $y$ \\
\hline 226980 & 61 & 1 & 389018 & 73 & 1 & 592705 & 84 & 1 & 884737 & 96 & 1 & 1225042 & 107 & 1 \\
\hline 226982 & 61 & & 91592 & 4097 & 56 & 596239 & 505 & 6 & 87042 & 1153 & 12 & 1225044 & 107 & 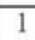 \\
\hline 238327 & 62 & 1 & 405223 & 74 & 1 & 599788 & 253 & 3 & 889352 & 577 & 6 & \begin{tabular}{|l}
1242297 \\
\end{tabular} & 215 & 2 \\
\hline 238329 & 62 & 1 & 405225 & 74 & 1 & 603351 & 169 & 2 & 891666 & 385 & 4 & 1248084 & 323 & 3 \\
\hline 246099 & 188 & 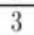 & 416275 & 224 & 3 & 614124 & 85 & 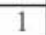 & 893984 & 289 & 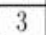 & \begin{tabular}{|l}
125889 \\
\end{tabular} & 647 & 6 \\
\hline 250046 & 63 & 1 & 418509 & 374 & 5 & 614126 & 85 & 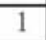 & 898632 & 193 & 2 & 1255828 & 971 & 9 \\
\hline 250048 & 63 & 1 & 420751 & 1124 & 15 & 636055 & 86 & 1 & 902629 & 1353 & 14 & 1257769 & 1943 & 18 \\
\hline 254037 & 190 & 3 & 421874 & 75 & 1 & 636057 & 86 & 1 & 912672 & 97 & 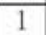 & \begin{tabular}{|l|l}
1259711 \\
\end{tabular} & 108 & 1 \\
\hline 256048 & 127 & 2 & 421876 & 75 & 1 & 650963 & 260 & 3 & 912674 & 97 & 1 & 1259713 & 108 & 1 \\
\hline 259084 & 255 & 4 & 423001 & 1126 & 15 & 658502 & 87 & 1 & 937082 & 685 & 7 & 1261657 & 1945 & 18 \\
\hline 260611 & 511 & 8 & 425259 & 376 & 5 & 658504 & 87 & 1 & 941191 & 98 & 1 & 1263604 & 973 & 9 \\
\hline 262143 & 64 & 1 & 427525 & 226 & 3 & 666101 & 262 & 3 & 941193 & 98 & 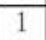 & 1265553 & 649 & 6 \\
\hline 262145 & 64 & 1 & 430369 & 151 & 2 & 669922 & 175 & 2 & 945314 & 687 & 7 & 1271412 & 325 & 3 \\
\hline 263683 & 513 & 8 & 438975 & 76 & 1 & 681471 & 88 & 1 & 960531 & 296 & 3 & 1277289 & 217 & 2 \\
\hline 265228 & 257 & 4 & 438977 & 76 & 1 & 681473 & 88 & 1 & 970298 & 99 & 1 & 1295028 & 109 & 1 \\
\hline 268336 & 129 & 2 & 447697 & 153 & 2 & 693154 & 177 & 2 & 970300 & 99 & 1 & 1295030 & 109 & 1 \\
\hline 274624 & 65 & 1 & 456532 & 77 & 1 & 704968 & 89 & 1 & 980133 & 298 & 3 & 1306421 & 9948 & 91 \\
\hline 274626 & 65 & 1 & 456534 & 77 & 1 & 704970 & 89 & 1 & 980838 & 1391 & 14 & 1330999 & 110 & 1 \\
\hline 283162 & 197 & 0 & 468494 & 233 & 3 & 710467 & 1160 & 13 & 985075 & 199 & 2 & 1331001 & 110 & 1 \\
\hline 287495 & 66 & 1 & 474551 & 78 & 1 & 712306 & 1161 & 13 & 994012 & 499 & 5 & 1355347 & 332 & 3 \\
\hline 287497 & 66 & 1 & 474553 & 78 & 1 & 720930 & 269 & 3 & 997003 & 999 & 10 & 1367630 & 111 & 1 \\
\hline 291874 & 199 & 3 & 480662 & 235 & 3 & 728999 & 90 & 1 & 999999 & 100 & 1 & 1367632 & 111 & 1 \\
\hline 300762 & 67 & 1 & \begin{tabular}{|l}
493038 \\
\end{tabular} & 79 & 1 & 729001 & 90 & 1 & 1000001 & 100 & 1 & 1379989 & 334 & 3 \\
\hline 300764 & 67 & 1 & 493040 & 79 & 1 & 737130 & 271 & 3 & 1003003 & 1001 & 10 & 1386196 & 223 & 2 \\
\hline 307547 & 135 & 2 & 502460 & 159 & 2 & 753570 & 91 & 1 & 1006012 & 501 & 5 & 1395541 & 447 & 4 \\
\hline 314431 & 68 & 1 & \begin{tabular}{|l}
506115 \\
\end{tabular} & 1036 & 13 & 753572 & 91 & 1 & 1015075 & 201 & 2 & 1404927 & 112 & 1 \\
\hline 314433 & 68 & 1 & 507215 & 319 & 4 & 766061 & 183 & 2 & 1017241 & 704 & 7 & 1404929 & 112 & 1 \\
\hline 321419 & 137 & 2 & 507582 & 1037 & 13 & 778687 & 92 & 1 & 1021582 & 705 & 7 & 1414357 & 449 & 4 \\
\hline 323771 & 206 & 3 & 511999 & 80 & 1 & 778689 & 92 & 1 & 1030300 & 101 & 1 & 1423828 & 225 & 2 \\
\hline 328508 & 69 & 1 & 512001 & 80 & 1 & 791453 & 185 & 2 & 1030302 & 101 & 1 & 1442896 & 113 & 1 \\
\hline 328510 & 69 & 1 & 516815 & 321 & 4 & 795739 & 278 & 3 & 1050838 & 305 & 3 & 1442898 & 113 & 1 \\
\hline 333293 & 208 & 3 & 521660 & 161 & 2 & 804356 & 93 & 1 & 1061207 & 102 & 1 & 1468586 & 341 & 3 \\
\hline 342999 & 70 & 1 & \begin{tabular}{|l}
524907 \\
\end{tabular} & 242 & 3 & \begin{tabular}{|l}
804358 \\
\end{tabular} & 93 & 1 & 1061209 & 102 & 1 & 1481543 & 114 & 1 \\
\hline 343001 & 70 & 1 & 529257 & 728 & 9 & 813037 & 280 & 3 & 1071646 & 307 & 3 & 1481545 & 114 & 1 \\
\hline 356057 & 4040 & 57 & 531440 & 81 & 1 & 830583 & 94 & 1 & 1092726 & 103 & 1 & 1494578 & 343 & 3 \\
\hline 357910 & 71 & 1 & 531442 & 81 & 1 & 830585 & 94 & 1 & 1092728 & 103 & 1 & 1520874 & 115 & 1 \\
\hline 357912 & 71 & 1 & 533631 & 730 & 9 & 857374 & 95 & 1 & 1108718 & 207 & 2 & 1520876 & 115 & 1 \\
\hline 365526 & 143 & 2 & 538029 & 244 & 3 & 857376 & 95 & 1 & 1124863 & 104 & 1 & 1530341 & 2420 & 21 \\
\hline 368088 & 215 & 3 & 551367 & 82 & 1 & 865134 & 667 & 7 & 1124865 & 104 & 1 & 1540799 & 231 & 2 \\
\hline 370662 & 431 & 6 & 551369 & 82 & 1 & 869031 & 668 & 7 & 1141166 & 209 & 2 & 1560895 & 116 & 1 \\
\hline 373247 & 72 & 1 & 571786 & 83 & 1 & 870984 & 191 & 2 & 1146635 & 314 & 3 & 1560897 & 116 & 1 \\
\hline 373249 & 72 & 1 & 571788 & 83 & 1 & 875552 & 287 & 3 & 1157624 & 105 & 1 & 1581167 & 233 & 2 \\
\hline 375846 & 433 & 6 & 582183 & 167 & 2 & 877842 & 383 & 4 & 1157626 & 105 & 1 & 1587963 & 350 & 3 \\
\hline 378456 & 217 & 3 & 585676 & 251 & 3 & 880136 & 575 & 6 & 1168685 & 316 & 3 & 1601612 & 117 & 1 \\
\hline 381078 & 145 & 2 & 589183 & 503 & 6 & 882434 & 1151 & 12 & 1191015 & 106 & 1 & 1601614 & 117 & 1 \\
\hline 389016 & 73 & 1 & 592703 & 84 & 1 & 884735 & 96 & 1 & 1191017 & 106 & 1 & 1615341 & 352 & 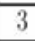 \\
\hline
\end{tabular}


Solving Binomial Thue Equations

\begin{tabular}{|c|c|c|c|c|c|c|c|c|c|c|c|c|c|c|}
\hline$m$ & $x$ & & & & & & & & $\ldots$ & & & $m$ & $x$ & \\
\hline 430 & & & & & & & 424 & & 00 & & 15 & $2=15$ & 100 & 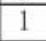 \\
\hline & & & & & & & & & & & & & 162 & 1 \\
\hline & 19 & 1 & 6690 & 29 & & 63289 & 142 & & 75001 & 50 & & 60282 & 1459 & 9 \\
\hline & & 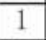 & & & & & 54 & & & & 15 & & 487 & 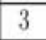 \\
\hline & 51 & & & 26 & & & 43 & & 18 & 51 & & 265 & 841 & 42 \\
\hline 66490 & 239 & 2 & 96999 & 130 & & 24208 & 143 & & 397550 & 451 & 3 & 330746 & 163 & 1 \\
\hline & 9 & 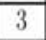 & & & & & or & & & 151 & & & 100 & 1 \\
\hline & 19 & 6 & & 31 & & & 31 & & 952 & 11 & & 70723 & 327 & 2 \\
\hline 27999 & 120 & 1 & 48092 & 131 & & 70459 & 575 & & 477266 & 303 & 2 & 410943 & 164 & 1 \\
\hline & 120 & 1 & & & & & 863 & & & 152 & & & 164 & 1 \\
\hline 5210 & 21 & $\rho_{1}$ & 88 & & & & 27 & & & 52 & & 331 & 3448 & 21 \\
\hline 2440 & 361 & 3 & 91267 & 791 & & 85983 & 144 & & 546578 & 305 & & 151411 & 329 & 2 \\
\hline & 241 & 2 & 67 & 132 & & & 144 & & & 58 & & 155 & 94 & 3 \\
\hline & 30 & , & 89 & 132 & & & 729 & & & 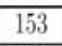 & & 92124 & 65 & 1 \\
\hline 1560 & 121 & 1 & 8691 & 793 & & 34 & 865 & & 881578 & 153 & & 492126 & 165 & 1 \\
\hline 62 & 21 & 1 & & 397 & & & 577 & & & 60 & & 05 & 496 & 3 \\
\hline 5557 & 32 & 11 & 03 & 265 & & & 1010 & & 63 & 7 & & 95 & 166 & 1 \\
\hline 847 & 122 & 1 & 2636 & 133 & & 38 & 433 & & 652265 & 54 & & 774297 & 166 & 1 \\
\hline 49 & 122 & 1 & 2638 & 133 & & & 1011 & & 74 & 155 & & 62 & 167 & 1 \\
\hline 779 & 68 & 3 & 61 & 134 & & & 28 & & 723876 & -5 & & 0.0 & 167 & 1 \\
\hline 866 & 23 & 1 & 66105 & 134 & & 4 & 145 & & 760029 & 311 & & 699422 & 335 & 2 \\
\hline 0868 & 123 & 1 & 2195 & 404 & & & 145 & & 152 & 67 & & 13464 & 50 & 3 \\
\hline & 370 & 3 & 43. & 121 & & & 14 & & 784261 & 935 & & 27534 & 100 & 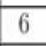 \\
\hline & 247 & 2 & 74 & 135 & & 37 & 146 & & 796415 & 156 & 1 & 741631 & 168 & 1 \\
\hline & 24 & 1 & & 135 & & & 4097 & & 17 & 156 & & 5 & 16 & 1 \\
\hline & 24 & 1 & 36455 & 121 & & & 5126 & & 3808597 & 937 & & 755758 & 100 & 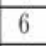 \\
\hline 2636 & 33 & 我 & 45 & 406 & & 3 & 440 & & 820804 & 469 & 3 & 769912 & 505 & 3 \\
\hline & 249 & 2 & & 271 & & & 1028 & & & 313 & & 94 & 337 & 2 \\
\hline & 24 & 5 & & 136 & & & 3086 & & 3869892 & 157 & & 1820221 & 2196 & 13 \\
\hline 124 & 125 & 1 & 15457 & 136 & & 2 & 147 & & 869894 & 157 & 7 & 826808 & 169 & 1 \\
\hline & 125 & 1 & 3302 & 273 & & & 147 & & 944311 & 15 & & 326810 & 169 & 1 \\
\hline 515 & 26 & 5 & 1352 & 137 & & & 3088 & & 3944313 & 158 & 1 & 1833403 & 2198 & 13 \\
\hline & 7 & 3 & & 137 & & & 1030 & & 994451 & 476 & & 912999 & 170 & 1 \\
\hline & 126 & 1 & 19074 & 41 & & & 442 & & 4019 & 159 & 1 & 1 & 170 & 1 \\
\hline 377 & 126 & 1 & 0.0011 & 138 & & & 295 & & 4019680 & 159 & 1 & 4931602 & 2383 & 14 \\
\hline & 379 & 3 & & 138 & & & 148 & & & 478 & & 4971027 & 512 & 3 \\
\hline & 127 & 1 & 71 & 41 & & 3 & 148 & & 4057720 & 319 & 2 & 000210 & 171 & 1 \\
\hline 8384 & 127 & 1 & 0010 & 139 & & 66062 & 2819 & & 4076830 & 639 & 4 & 5000212 & 171 & +2 \\
\hline & 255 & 2 & & 139 & 1 & & 2820 & 20 & 4095999 & 160 & 1 & 5029509 & 514 & 3 \\
\hline & 511 & 4 & & 27 & 3 & 99 & 297 & & 4096001 & 160 & 1 & $\overline{044201}$ & $\overline{343}$ & 2 \\
\hline 2091014 & 1023 & 8 & 2743999 & 140 & & and & 149 & & 4115230 & 641 & 4 & 5088447 & 172 & 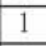 \\
\hline & 128 & 1 & & 140 & 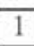 & & 149 & & \begin{tabular}{|l|l}
4134520 \\
\end{tabular} & 321 & 2 & 5088449 & 172 & 1 \\
\hline & & 1 & & 28 & . & 54 & 1047 & & 4173280 & 161 & 1 & 132953 & 345 & 2 \\
\hline & 1020 & 8 & 21000001 & 422 & & 3352550 & 449 & & 4173282 & 161 & 1 & 5177716 & 173 & - \\
\hline & 513 & 4 & 2000200 & 141 & 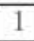 & & 1048 & 1 & 4225 & 485 & 3 & 5177718 & 173 & 1 \\
\hline 2121824 & 257 & 2 & 2803222 & 141 & 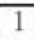 & $\overline{3361}$ & 749 & & 4242786 & 1457 & 9 & 5237806 & 521 & 3 \\
\hline
\end{tabular}




\begin{tabular}{|c|c|c|c|c|c|c|c|c|c|c|c|}
\hline$m$ & $x$ & $y$ & $m$ & $x$ & $y$ & $m$ & $x$ & $y$ & & & \\
\hline 5268023 & 174 & 1 & 6434855 & 186 & 1 & 7513084 & 1371 & 7 & & & \\
\hline 5268025 & 174 & 1 & 6434857 & 186 & 1 & 7521307 & 2743 & 14 & & & \\
\hline 5298358 & 523 & 3 & 6469514 & 559 & 3 & \begin{tabular}{|l}
7529535 \\
\end{tabular} & 196 & 1 & & & \\
\hline 5341021 & 874 & 5 & 6539202 & 187 & 1 & 7529537 & 196 & 1 & & & \\
\hline 5359374 & 175 & 1 & 6539204 & 187 & 1 & \begin{tabular}{|l}
7537771 \\
\end{tabular} & 2745 & 14 & $m$ & $x$ & $y$ \\
\hline 5359376 & 175 & 1 & 6591797 & 375 & 2 & \begin{tabular}{|l|}
7546012 \\
\end{tabular} & 1373 & 7 & 8741815 & 206 & 1 \\
\hline 5377771 & 876 & 5 & 6644671 & 188 & 1 & 7587307 & 393 & 2 & 8741817 & 206 & 1 \\
\hline 5405444 & 351 & 2 & 6644673 & 188 & 1 & \begin{tabular}{|l|}
7645372 \\
\end{tabular} & 197 & 1 & 8826963 & 620 & 3 \\
\hline 5428577 & 703 & 4 & 6697829 & 377 & 2 & \begin{tabular}{|l|}
7645374 \\
\end{tabular} & 197 & 1 & 8869742 & 207 & 1 \\
\hline 5451775 & 176 & 1 & 6715611 & 566 & 3 & 7723254 & 593 & 3 & 8869744 & 207 & 1 \\
\hline 5451777 & 176 & 1 & 6739369 & 1700 & 9 & \begin{tabular}{|l|}
7762391 \\
\end{tabular} & 198 & 1 & 8912661 & 622 & 3 \\
\hline 5475041 & 705 & 4 & 6751268 & 189 & 1 & \begin{tabular}{|l}
7762393 \\
\end{tabular} & 198 & 1 & 8934172 & 415 & 2 \\
\hline 5498372 & 353 & 2 & 6751270 & 189 & 1 & 7801662 & 595 & 3 & 8966503 & 831 & 4 \\
\hline 5513963 & 530 & 3 & 6763183 & 1702 & 9 & \begin{tabular}{|l}
7829793 \\
\end{tabular} & 1390 & 7 & 8998911 & 208 & 1 \\
\hline 5545232 & 177 & 1 & 6787053 & 568 & 3 & 7846704 & 1391 & 7 & 8998913 & 208 & 1 \\
\hline 5545234 & 177 & 1 & 6858999 & 190 & 1 & \begin{tabular}{|l|}
7880598 \\
\end{tabular} & 199 & 1 & 9031399 & 833 & 4 \\
\hline 5576621 & 532 & 3 & 6859001 & 190 & 1 & \begin{tabular}{|l}
7880600 \\
\end{tabular} & 199 & 1 & 9063964 & 417 & 2 \\
\hline 5639751 & 178 & 1 & 6967870 & 191 & 1 & \begin{tabular}{|l}
7926126 \\
\end{tabular} & 93311 & 468 & 9114217 & 5431 & 26 \\
\hline 5639753 & 178 & 1 & 6967872 & 191 & 1 & 7940150 & 399 & 2 & 9129328 & 209 & 1 \\
\hline 5712483 & 3754 & 21 & 7022736 & 383 & 2 & 7976024 & 999 & 5 & 9129330 & 209 & 1 \\
\hline 5735338 & 179 & 1 & 7041088 & 575 & 3 & \begin{tabular}{|l|}
7988006 \\
\end{tabular} & 1999 & 10 & 9216970 & 629 & 3 \\
\hline 5735340 & 179 & 1 & 7050276 & 767 & 4 & 7999999 & 200 & 1 & 9260999 & 210 & 1 \\
\hline 5783535 & 359 & 2 & 7059472 & 1151 & 6 & 8000001 & 200 & 1 & 9261001 & 210 & 1 \\
\hline 5799660 & 539 & 3 & 7064073 & 1535 & 8 & 8012006 & 2001 & 10 & 9305170 & 631 & 3 \\
\hline 5815815 & 1079 & 6 & 7068676 & 2303 & 12 & 8024024 & 1001 & 5 & 9393930 & 211 & 1 \\
\hline 5831999 & 180 & 1 & 7073281 & 4607 & 24 & 8060150 & 401 & 2 & 9393932 & 211 & 1 \\
\hline 5832001 & 180 & 1 & 7077887 & 192 & 1 & 8080267 & 602 & 3 & 9460871 & 423 & 2 \\
\hline 5848215 & 1081 & 6 & 7077889 & 192 & 1 & 8120600 & 201 & 1 & 9528127 & 212 & 1 \\
\hline 5864460 & 541 & 3 & 7082497 & 4609 & 24 & \begin{tabular}{|l|}
8120602 \\
\end{tabular} & 201 & 1 & 9528129 & 212 & 1 \\
\hline 5880735 & 361 & 2 & 7087108 & 2305 & 12 & 8161069 & 604 & 3 & 9595703 & 425 & 2 \\
\hline 5929740 & 181 & 1 & 7091721 & 1537 & 8 & 8242407 & 202 & 1 & 9603769 & 17855 & 84 \\
\hline 5929742 & 181 & 1 & 7096336 & 1153 & 6 & 8242409 & 202 & 1 & 9606402 & 4039 & 19 \\
\hline 6028567 & 182 & 1 & 7105572 & 769 & 4 & 8341522 & 6287 & 31 & 9613539 & 4040 & 19 \\
\hline 6028569 & 182 & 1 & 7114816 & 577 & 3 & 8345503 & 6288 & 31 & 9618299 & 638 & 3 \\
\hline 6095059 & 548 & 3 & 7133328 & 385 & 2 & 8365426 & 203 & 1 & 9663596 & 213 & 1 \\
\hline 6128486 & 183 & 1 & 7189056 & 193 & 1 & 8365428 & 203 & 1 & 9663598 & 213 & 1 \\
\hline 6128488 & 183 & 1 & 7189058 & 193 & 1 & 8427393 & 407 & 2 & 9709037 & 640 & 3 \\
\hline 6162037 & 550 & 3 & 7221032 & 1353 & 7 & 8448116 & 611 & 3 & 9800343 & 214 & 1 \\
\hline 6178858 & 367 & 2 & 7237055 & 1354 & 7 & 8468873 & 1223 & 6 & 9800345 & 214 & 1 \\
\hline 6229503 & 184 & 1 & 7301383 & 194 & 1 & \begin{tabular}{|l|l|}
8489663 \\
\end{tabular} & 204 & 1 & 9938374 & 215 & 1 \\
\hline 6229505 & 184 & 1 & 7301385 & 194 & 1 & 8489665 & 204 & 1 & 9938376 & 215 & 1 \\
\hline 6280426 & 369 & 2 & 7376915 & 584 & 3 & 8510489 & 1225 & 6 & & & \\
\hline 6331624 & 185 & 1 & 7414874 & 195 & 1 & 8531348 & 613 & 3 & & & \\
\hline 6331626 & 185 & 1 & 7414876 & 195 & 1 & 8552241 & 409 & 2 & & & \\
\hline 6400322 & 557 & 3 & 7452965 & 586 & 3 & 8615124 & 205 & 1 & & & \\
\hline 6402292 & 6499 & 35 & 7472059 & 391 & 2 & 8615126 & 205 & 1 & & & \\
\hline
\end{tabular}


4.2. Solutions for $n=4$

\begin{tabular}{|c|c|c|c|c|c|c|c|c|c|c|c|}
\hline$m$ & $x$ & $y$ & $m$ & $x$ & $y$ & $m$ & $x$ & $y$ & $m$ & $x$ & $y$ \\
\hline 2 & 1 & 1 & 65535 & 16 & 1 & 707282 & 29 & 1 & 3580610 & 87 & 2 \\
\hline 5 & 3 & 2 & 65537 & 16 & 1 & 757335 & 59 & 2 & 3748095 & 44 & 1 \\
\hline 15 & 2 & 1 & 69729 & 65 & 4 & 809999 & 30 & 1 & 3748097 & 44 & 1 \\
\hline 17 & 2 & 1 & 74120 & 33 & 2 & 810001 & 30 & 1 & 3851367 & 443 & 10 \\
\hline 39 & 5 & 2 & 83520 & 17 & 1 & 865365 & 61 & 2 & 3921390 & 89 & 2 \\
\hline 80 & 3 & 1 & 83522 & 17 & 1 & 923520 & 31 & 1 & 4100624 & 45 & 1 \\
\hline 82 & 3 & 1 & 93789 & 35 & 2 & 923522 & 31 & 1 & 4100626 & 45 & 1 \\
\hline 150 & 7 & 2 & 104975 & 18 & 1 & 984560 & 63 & 2 & 4285935 & 91 & 2 \\
\hline 255 & 4 & 1 & 104977 & 18 & 1 & 1016190 & 127 & 4 & 4477455 & 46 & 1 \\
\hline 257 & 4 & 1 & 114240 & 239 & 13 & 1048575 & 32 & 1 & 4477457 & 46 & 1 \\
\hline 410 & 9 & 2 & 117135 & 37 & 2 & 1048577 & 32 & 1 & 4675325 & 93 & 2 \\
\hline 624 & 5 & 1 & 130320 & 19 & 1 & 1081730 & 129 & 4 & 4879680 & 47 & 1 \\
\hline 626 & 5 & 1 & 130322 & 19 & 1 & 1115664 & 65 & 2 & 4879682 & 47 & 1 \\
\hline 915 & 11 & 2 & 144590 & 39 & 2 & 1185920 & 33 & 1 & 5090664 & 95 & 2 \\
\hline 1295 & 6 & 1 & 159999 & 20 & 1 & 1185922 & 33 & 1 & 5198685 & 191 & 4 \\
\hline 1297 & 6 & 1 & 160001 & 20 & 1 & 1259445 & 67 & 2 & 5308415 & 48 & 1 \\
\hline 1785 & 13 & 2 & 176610 & 41 & 2 & 1336335 & 34 & 1 & 5308417 & 48 & 1 \\
\hline 2400 & 7 & 1 & 194480 & 21 & 1 & 1336337 & 34 & 1 & 5419875 & 193 & 4 \\
\hline 2402 & 7 & 1 & 194482 & 21 & 1 & 1416695 & 69 & 2 & 5533080 & 97 & 2 \\
\hline 3164 & 15 & 2 & 213675 & 43 & 2 & 1500624 & 35 & 1 & 5764800 & 49 & 1 \\
\hline 4095 & 8 & 1 & 234255 & 22 & 1 & 1500626 & 35 & 1 & 5764802 & 49 & 1 \\
\hline 4097 & 8 & 1 & 234257 & 22 & 1 & 1588230 & 71 & 2 & 6003725 & 99 & 2 \\
\hline 5220 & 17 & 2 & 256289 & 45 & 2 & 1679615 & 36 & 1 & 6249999 & 50 & 1 \\
\hline 6560 & 9 & 1 & 279840 & 23 & 1 & 1679617 & 36 & 1 & 6250001 & 50 & 1 \\
\hline 6562 & 9 & 1 & 279842 & 23 & 1 & 1755519 & 182 & 5 & 6503775 & 101 & 2 \\
\hline 7140 & 239 & 26 & 304980 & 47 & 2 & 1774890 & 73 & 2 & 6765200 & 51 & 1 \\
\hline 8145 & 19 & 2 & 331775 & 24 & 1 & 1874160 & 37 & 1 & 6765202 & 51 & 1 \\
\hline 9999 & 10 & 1 & 331777 & 24 & 1 & 1874162 & 37 & 1 & 7034430 & 103 & 2 \\
\hline 10001 & 10 & 1 & 360300 & 49 & 2 & 1977539 & 75 & 2 & 7311615 & 52 & 1 \\
\hline 12155 & 21 & 2 & 390624 & 25 & 1 & 2085135 & 38 & 1 & 7311617 & 52 & 1 \\
\hline 14640 & 11 & 1 & 390626 & 25 & 1 & 2085137 & 38 & 1 & 7596914 & 105 & 2 \\
\hline 14642 & 11 & 1 & 422825 & 51 & 2 & 2197065 & 77 & 2 & 7890480 & 53 & 1 \\
\hline 17490 & 23 & 2 & 456975 & 26 & 1 & 2313440 & 39 & 1 & 7890482 & 53 & 1 \\
\hline 20735 & 12 & 1 & 456977 & 26 & 1 & 2313442 & 39 & 1 & 8192475 & 107 & 2 \\
\hline 20737 & 12 & 1 & 493155 & 53 & 2 & 2434380 & 79 & 2 & 8295040 & 161 & 3 \\
\hline 24414 & 25 & 2 & 505679 & 80 & 3 & 2559999 & 40 & 1 & 8398565 & 323 & 6 \\
\hline 28560 & 13 & 1 & 518440 & 161 & 6 & 2560001 & 40 & 1 & 8503055 & 54 & 1 \\
\hline 28562 & 13 & 1 & 531440 & 27 & 1 & 2690420 & 81 & 2 & 8503057 & 54 & 1 \\
\hline 33215 & 27 & 2 & 531442 & 27 & 1 & 2825760 & 41 & 1 & 8608519 & 325 & 6 \\
\hline 38415 & 14 & 1 & 544685 & 163 & 6 & 2825762 & 41 & 1 & 8714960 & 163 & 3 \\
\hline 38417 & 14 & 1 & 558175 & 82 & 3 & 2966145 & 83 & 2 & 8822385 & 109 & 2 \\
\hline 44205 & 29 & 2 & 571914 & 55 & 2 & 3111695 & 42 & 1 & 9150624 & 55 & 1 \\
\hline 50624 & 15 & 1 & 614655 & 28 & 1 & 3111697 & 42 & 1 & 9150626 & 55 & 1 \\
\hline 50626 & 15 & 1 & 614657 & 28 & 1 & 3262539 & 85 & 2 & 9487940 & 111 & 2 \\
\hline 57720 & 31 & 2 & 659750 & 57 & 2 & 3418800 & 43 & 1 & 9834495 & 56 & 1 \\
\hline 61535 & 63 & 4 & 707280 & 29 & 1 & 3418802 & 43 & 1 & 9834497 & 56 & 1 \\
\hline
\end{tabular}


4.3. Solutions for $n=5$

\begin{tabular}{|c|c|c|c|c|c|c|c|c|c|c|c|}
\hline$m$ & & 9 & $m$ & $\omega$ & & $m$ & $x$ & & & & \\
\hline 2 & & 1 & 32767 & 8 & & 537825 & 14 & & $m$ & $x$ & \\
\hline 31 & & & 2769 & 0 & & 759374 & 15 & 1 & 3199999 & 0 & \\
\hline 33 & & & 0 & $c$ & & 759: & 19 & & 3200001 & 20 & \\
\hline 242 & & 1 & 59050 & 9 & & & 31 & $?$ & 4084100 & 21 & 1 \\
\hline 244 & : & 1 & 99 & 10 & 1 & 75 & $16 \quad$ & - & 4084102 & 21 & \\
\hline 1023 & 4 & 1 & 100 & & & & It & & 5153631 & 22 & \\
\hline 1025 & 2 & & & & & & & & 5153633 & 22 & \\
\hline 3124 & 5 & & & 11 & & & & & 6436342 & 23 & \\
\hline 3126 & 3 & & i) & & & & 17 & & 6436344 & 23 & \\
\hline 7775 & 6 & & 248833 & & & 67 & 1 & & 7962623 & 24 & \\
\hline 7777 & 6 & 1 & 371292 & 13 & & 09 & 10 & & 7962625 & 24 & \\
\hline 16806 & 7 & 1 & 371294 & 13 & 1 & 247 & 19 & 1 & 9765624 & 25 & \\
\hline 16808 & 7 & & 537823 & 14 & & 2476100 & 19 & & 9765626 & 25 & 1 \\
\hline
\end{tabular}

4.4. Solutions for $n=7$

\begin{tabular}{|c|c|c|}
\hline$m$ & $x$ & $y$ \\
\hline 2 & 1 & 1 \\
\hline 127 & 2 & 1 \\
\hline 129 & 2 & 1 \\
\hline 2186 & 3 & 1 \\
\hline 2188 & 3 & 1 \\
\hline 16383 & 4 & 1 \\
\hline 16385 & 4 & 1 \\
\hline 78124 & 5 & 1 \\
\hline 78126 & 5 & 1 \\
\hline 279935 & 6 & 1 \\
\hline 279937 & 6 & 1 \\
\hline 823542 & 7 & 1 \\
\hline 823544 & 7 & 1 \\
\hline 2097151 & 8 & 1 \\
\hline 2097153 & 8 & 1 \\
\hline 4782968 & 9 & 1 \\
\hline 4782970 & 9 & 1 \\
\hline 9999999 & 10 & 1 \\
\hline
\end{tabular}


4.5. Solutions for $n=11$

\begin{tabular}{|c|c|c|}
\hline$m$ & $x$ & $y$ \\
\hline 2 & 1 & 1 \\
\hline 2047 & 2 & 1 \\
\hline 2049 & 2 & 1 \\
\hline 177146 & 3 & 1 \\
\hline 177148 & 3 & 1 \\
\hline 4194303 & 4 & 1 \\
\hline 4194305 & 4 & 1 \\
\hline
\end{tabular}

4.6. Solutions for $n=13$

\begin{tabular}{|c|c|c|}
\hline$m$ & $x$ & $y$ \\
\hline 2 & 1 & 1 \\
\hline 8191 & 2 & 1 \\
\hline 8193 & 2 & 1 \\
\hline 1594322 & 3 & 1 \\
\hline 1594324 & 3 & 1 \\
\hline
\end{tabular}

4.7. Solutions for $n=17$

\begin{tabular}{|c|c|c|}
\hline$m$ & $x$ & $y$ \\
\hline 2 & 1 & 1 \\
\hline 131071 & 2 & 1 \\
\hline 131073 & 2 & 1 \\
\hline
\end{tabular}

4.8. Solutions for $n=19$

\begin{tabular}{|c|c|c|}
\hline$m$ & $x$ & $y$ \\
\hline 2 & 1 & 1 \\
\hline 524287 & 2 & 1 \\
\hline 524289 & 2 & 1 \\
\hline
\end{tabular}


4.9. Solutions for $n=23$

\begin{tabular}{|c|c|c|}
\hline$m$ & $x$ & $y$ \\
\hline 2 & 1 & 1 \\
\hline 8388607 & 2 & 1 \\
\hline 8388609 & 2 & 1 \\
\hline
\end{tabular}

4.10. Solutions for $n=29$

\begin{tabular}{|l|l|l|}
\hline$m$ & $x$ & $y$ \\
\hline 2 & 1 & 1 \\
\hline
\end{tabular}

References

[1] A. Bazsó, A. Bérczes, K. Győry and Á. Pintér, On the resolution of equations $A x^{n}-B y^{n}=C$ in integers $x, y$ and $n \geq 3$, II, Publ. Math. (Debrecen), 76 (2010), 227-250.

[2] M. A. Bennett, Rational approximation to algebraic numbers of small height: The Diophantine equation $\left|a x^{n}-b y^{n}\right|=1$, J. Reine Angew. Math. 535 (2001), 1-49.

[3] B. W. Char, K. O. Geddes, G. H. Gonnet, M. B. Monagan and S. M. Watt, (eds.) MAPLE, Reference Manual, Watcom Publications, Waterloo, Canada, 1988.

[4] I. Gaál, Diophantine Equations and Power Integral Bases, Boston, Birkhäuser, 2002.

[5] I. Gaál and L. Remete, Binomial Thue equations and power integral bases in pure quartic fields, JP Journal of Algebra, Number Theory and Applications 32(1) (2014), 49-61.

[6] K. Győry and Á. Pintér, Binomial Thue equations, ternary equations, and power values of polynomials, J. Math. Sciences 180 (2012), 569-580.

[7] A. Pethő, On the resolution of Thue inequalities, J. Symbolic Comput. 4 (1987), 103-109. 